\title{
ENTRETIEN
}

\section{Les approches plurielles dans l'enseignement des langues: un entretien avec Michel Candelier}

\author{
Dr. Xavier Pascual Calvo \\ Universitat Autònoma de Barcelona
}

(Reçue le 29 Novembre 2011, version finale reçue le 19 Décembre 2011)

Michel Candelier est professeur à Université du Maine, Le Mans, France. Il est coordinateur d'un projet intitulé CARAP "Cadre de référence pour les approches plurielles", mené dans le cadre du programme "Valoriser les professionnels en langues » du Centre européen pour les langues vivantes (CELV) et a publié de nombreux articles à propos de l'enseignement à l'aide des approches plurielles visant à développer des compétences plurilingues et interculturelles chez les apprenants de toutes disciplines.

INTERVIEWER: M. Candelier, on constate un travail important de la part du Conseil de l'Europe vis à vis de la promotion de la compétence plurilingue et interculturelle, mais où en sommes-nous de l'éducation plurilingue et pluriculturelle ? Quel est d'après vous le chemin à suivre pour développer cette sorte de compétences dans nos systèmes éducatifs?

MICHEL CANDELIER: Le souhaitable dont nous parlons ici, c'est ce que l'on peut / doit souhaiter atteindre par / dans l'enseignement en ce qui concerne les langues. Bien sûr, cela varie forcément en fonction des contextes et des niveaux scolaires. Mais il est sans doute possible d'énoncer quelques principes généraux en ce qui concerne ce qui devrait être atteint et pour quelles langues et la façon de procéder pour l’atteindre.

À mon avis, il ne faut pas se limiter pas à ce que beaucoup d'enseignants font aujourd'hui, avec les contraintes qui sont les leurs. Il se peut bien sûr que l'on réalise déjà une partie de ce qui est selon eux souhaitable, mais il est vraisemblable aussi que certains rencontrent certains freins ou obstacles : organisation des enseignements, programmes, examens, traditions didactiques, horaires, nombre d'élèves...Il ne faut pas non plus décrire un monde utopique où toutes les contraintes disparaitraient comme par enchantement: sauf situation exceptionnelle, les élèves ne peuvent pas faire 25 heures de langue(s) par semaine, dans des classes limitées à douze élèves maximum... 
Le souhaitable doit être réalisable au prix d'efforts raisonnablement envisageables. Le Conseil de l'Europe a déjà commencé et a développé des instruments particulièrement complets en matière de réflexions et de propositions pour la didactique des langues (en particulier, le Cadre européen commun de référence pour les langues - Conseil de l'Europe, 2001*) et les politiques linguistiques éducatives (en particulier, le Guide pour l'élaboration des politiques linguistiques en Europe, Beacco \& Byram, 2007).

INTERVIEWER: À quels principes sur les politiques linguistiques pour l'Europe faites vous reférence?

MICHEL CANDELIER: La détermination de principes pour les politiques linguistiques éducatives européennes se situe sur un continuum qui porte de choix techniques à des choix identitaires. Toutes ces options sont présentes à la fois dans le débat sur le devenir européen [...] : conception économique (circulation des biens et des personnes), culturelle, sociale (cohésion sociale), politique (démocratie et droits des minorités) et même anthropologique (quelle forme d'identité communautaire pour les Européens ?).

L'Europe n'est pas une entité politique de même nature qu'un État-nation, à qui il suffirait de se doter d'une (ou de plusieurs) langue(s) nationale(s) officielle(s), pour en tirer une forme d'unité ou d'identité. C'est un ensemble historique foncièrement original, espace pluriel, dans lequel ont cours des variétés linguistiques multiples, expression d'une diversité culturelle constitutive, qui se sont enrichies mutuellement, mais dans lequel aucune variété linguistique n’a occupé une position dominante dans la longue durée. Pour les citoyens européens, il n'existe probablement aucune langue qui puisse, à elle seule, représenter la langue d'appartenance à cet espace.

La détermination de principes pour les politiques linguistiques éducatives doit aussi tenir compte des évolutions en cours : d'un côté, l'internationalisation, la mise en marché de la plupart des activités humaines, le rôle croissant des entreprises multinationales, la fonction sociale modélisante de l'économie, l'impact de la culture télévisuelle, de l'autre, la nouvelle émergence de sentiments identitaires, le retour des nationalismes à base ethnocentrique.

INTERVIEWER: Et les approches plurielles?

MICHEL CANDELIER: L'éducation plurilingue et pluriculturelle joue actuellement un rôle central dans l'enseignement/apprentissage des langues vivantes. Elle a été particulièrement valorisée par les experts de la division des politiques linguistiques du Conseil de l'Europe. Le terme d'approches plurielles qui est aujourd'hui générique en englobe quatre: éveil aux langues, 
intercompréhension entre les langues, approches interculturelles et didactique intégrée. Il n’existait jusqu’à présent aucun référentiel recensant les savoirs, savoir-être et savoir-faire que ces quatre approches permettent de développer. C’est pour cette raison que des travaux ont été entrepris sous l'égide du Centre européen pour les langues vivantes à Graz afin d'élaborer un référentiel de compétences pour les approches plurielles et pluriculturelles - le CARAP, conçu pour compléter et perfectionner des outils existants tels que le CECR ou les Portfolios (PEL).

Les enseignants de langues vivantes peuvent avoir recours à ce référentiel pour : résoudre un « problème » lié à la pluralité culturelle et/ou linguistique, développer un projet touchant à la pluralité culturelle et/ou linguistique et pour analyser/comprendre une activité didactique concernant la pluralité culturelle ou linguistique.

Pour illustrer une des entrées possibles au CARAP, nous allons présenter aussi des activités de l'éveil aux langues à exploiter en classe de FLE, car c'est justement dans les domaines du plurilinguisme qui peut se jouer le défi de l'enseignement du français, tout en admettant que la diversification des langues ouvre de nouvelles perspectives.

INTERVIEWER: La didactique intégrée des langues, qui est vraisemblablement la plus connue des trois, vise à aider l'apprenant à établir des liens entre toutes les langues. Comment cela serait-il possible dans le cadre de l'enseignement primaire ou secondaire?

MICHEL CANDELIER: Il s'agit plutôt d'établir des liens entre un nombre limité de langues, celles dont on recherche l'apprentissage dans un cursus scolaire (qu'il vise de façon «classique» les mêmes compétences pour toutes les langues enseignées ou qu’il prévoie des «compétences partielles» pour certaines d'entre elles). Le but est alors de prendre appui sur la langue première (ou la langue de l'école) pour faciliter l'accès à une première langue étrangère, puis sur ces deux langues pour faciliter l'accès à une seconde langue étrangère (les appuis pouvant aussi se manifester en retour). C’est dans cette direction que militaient déjà les travaux de E. Roulet au tout début des années 1980. C'est aussi dans cette perspective que s’inscrivent aujourd'hui de nombreux travaux portant sur «l'allemand après l'anglais» en tant que langues étrangères (cf. les travaux portant sur les langues tertiaires). On la retrouve également dans certaines modalités d'éducation bilingue (ou plurilingue) qui ont le souci d'optimiser les relations entre les langues utilisées (et leur apprentissage) pour construire une véritable compétence plurilingue.

INTERVIEWER: Selon la définition qui a été donnée de l'éveil aux langues dans le cadre des projets européens récents qui ont permis de le développer plus largement, l'éveil aux langues porte sur des langues que l'école n’a pas l'ambition d’enseigner... 
MICHEL CANDELIER: En effet mais cela ne signifie pas que la démarche porte uniquement sur ces langues. Elle inclut également la langue de l'école et toute langue autre en cours d'apprentissage. Mais elle ne se limite pas à ces langues «apprises». Elle intègre toutes sortes d'autres variétés linguistiques, de la famille, de l'environnement ... et du monde, sans être exclusive d'aucun ordre. Par le nombre important de langues sur lesquelles les élèves sont amenés à travailler - plusieurs dizaines, le plus souvent - l'éveil aux langues peut apparaître comme une approche plurielle «extrême».

Conçu principalement comme accueil des élèves dans la diversité des langues (et de leurs langues!) dès le début de la scolarité, comme vecteur d'une meilleure reconnaissance dans le contexte scolaire des langues «apportées» par les élèves allophones, comme une sorte de propédeutique développée à l'école primaire, il peut également être promu comme accompagnement des apprentissages linguistiques tout le long de la scolarité.

INTERVIEWER: Un peu et passons maintenant si vous le voulez bien au rapport que vous établissez entre les approches plurielles et le développement de cette compétence plurilingue et pluriculturelle.

MICHEL CANDELIER: Le plus important d'après moi, c'est tenir compte du fait que ce qui est en jeu c'est l'abandon d'une vision «cloisonnante» de la / des compétence(s) des individus en matière de langues et de cultures, abandon qui découle logiquement de la façon dont la «compétence plurilingue et pluriculturelle» est conçue par le Cadre européen commun de référence comme une compétence qui ne consiste pas en «une collection de compétences à communiquer distinctes et séparées suivant les langues», mais bien en une «compétence plurilingue et pluriculturelle qui englobe l'ensemble du répertoire langagier à disposition».

Dans ce sens, on ne peut dire plus clairement que les approches plurielles, telles que définies cidessus, ont un rôle capital à jouer dans la construction de la «compétence plurilingue et pluriculturelle» de chacun. Car comment assurer que les «variétés» ne seront pas «abordées de manière isolée» si on s'en tient à des approches «singulières»?

INTERVIEWER: Autrement dit, si l'on pense que la compétence plurilingue est bien celle que les instruments du Conseil de l'Europe décrivent, et si on souhaite réellement donner un sens au principe de synergie recommandé, il convient, pour aider l'apprenant à construire et à enrichir continuellement sa propre compétence plurilingue en s'appuyant sans cesse sur ce qu'il connaît 
déjà, de l'amener à se constituer un arsenal de savoirs, savoir-faire et savoir-être. Comment pourrait-on aider l'apprenant?

MICHEL CANDELIER: Concernant les faits linguistiques et culturels en général, il s'agit d'aider l'apprenant à se construire un arsenal relevant de l'ordre du «trans»: «trans-linguistique», «trans-culturel». Il s'agit par ailleurs de faciliter l'appui sur des aptitudes acquises à propos de / dans une langue ou culture particulière (ou certains aspects d'une langue ou culture particulière) pour accéder plus facilement à une autre. Il s'agit alors d'un arsenal relevant de l'ordre de l'«inter»: «inter-linguistique», «inter-culturel».

De tels savoirs, savoir-faire et savoir-être ne peuvent, bien évidemment, être développés que lorsque la classe est le lieu d'un examen simultané et d'une mise en relation de plusieurs langues et de plusieurs cultures. C'est-à-dire, dans le cadre d'approches plurielles des langues et des cultures.

INTERVIEWER: De ce que vous me racontez, on pourrait conclure que les finalités que l'on peut énoncer à propos des approches plurielles sont strictement identiques à celles que visent les instruments centraux du Conseil de l'Europe en matière de langues que sont le Cadre européen commun de référence pour les langues et le Guide pour l'élaboration des politiques linguistiques éducatives en Europe.

MICHEL CANDELIER: Si nous pouvons nous permettre ce raccourci (et ce manque évident de modestie), il nous semble difficile de contester le bien-fondé de l'argumentation selon laquelle les approches plurielles constituent l'outil d'articulation indispensable de tous les efforts didactiques visant à faciliter le développement et l'enrichissement continu de la compétence plurilingue et pluriculturelle des individus apprenants.

Sans approches plurielles, c'est la formation plurilingue préconisée par le Guide pour l'élaboration des politiques linguistiques éducatives en Europe qui est compromise. Sans articulation entre les langues, tout effort visant à augmenter le nombre de langues apprises par un même individu-apprenant dans le cadre de l'éducation formelle se heurtera immédiatement à des limites en termes à la fois de capacité d'apprentissage et d'espace dans les curricula, limites que la synergie mise en œuvre par les approches plurielles permet d'élargir. Sans approches plurielles, c'est donc la diversité des langues proposées et apprises qui est réduite, c’est-à-dire la capacité de l'école à doter les apprenants des compétences linguistiques et culturelles diversifiées (et de la faculté à les étendre) dont chacun a besoin pour vivre, travailler, participer à la vie culturelle et démocratique dans un monde où la rencontre avec la diversité des langues et des 
cultures fait de plus en plus partie du quotidien, pour un nombre de plus en plus élevé d'individus.

INTERVIEWER: On peut déduire aussi que sans articulation entre les langues, ce sont aussi des pans entiers de l'expérience langagière antérieure des apprenants qui restent ignorés, c'est-àdire à la fois inexploités et, pour certaines langues, dévalorisés. N'est-ce pas?

MICHEL CANDELIER: $\quad \mathrm{Si}$, et il faut ajouter que par ce dernier terme - dévalorisés - nous abordons un second aspect des finalités des approches plurielles, que la perspective un peu technique de notre problématique première (approches plurielles et compétence plurilingue et pluriculturelle) ne nous avait pas fait rencontrer d'emblée: les approches plurielles, par la diversité des langues et des cultures au contact desquelles elles permettent de placer l'apprenant, sont un outil décisif pour la construction de ce que le Guide pour l'élaboration des politiques linguistiques éducatives en Europe appelle l’éducation au plurilinguisme.

INTERVIEWER: $\quad$ Si on dispose aujourd'hui de nombreux travaux, tant théoriques que pratiques, concernant chacune des diverses approches plurielles des langues et des cultures, il n’existe jusqu'à présent, hors de votre, projet aucun référentiel des savoirs, savoir-être et savoirfaire susceptibles d’être développés par des approches plurielles des langues et cultures.

MICHEL CANDELIER: En effet et l'absence d'un tel référentiel, pour un domaine de l'éducation aux langues et aux cultures dont nous venons de mettre en évidence le caractère central pour toute didactique visant la réalisation des buts et finalités fixés par le Conseil de l'Europe, constitue un handicap important pour l'enseignement-apprentissage des langues et cultures.

C'est ce qu'ont bien compris, désormais, certains concepteurs de curricula en développant une conception de l'éducation langagière incluant la diversité des approches plurielles et des enseignements de langues, voire d'autres disciplines. Les développements actuels des politiques éducatives en Suisse romande, au Val d'Aoste (cf. Cavalli 2005), en Andorre et en Catalogne en constituent de bons exemples .

INTERVIEWER: Ce cadre de référence pour les approches plurielles (CARAP) pour qui constituerait-il un outil intéressant?

MICHEL CANDELIER: Je pense à plusieurs usages... pour l'élaboration de curricula articulant, y compris dans la perspective d'une progression, l'acquisition des divers savoirs, savoir-être et savoir-faire auxquels les approches plurielles donnent (exclusivement / plus 
aisément) accès mais aussi pour l'articulation entre les approches plurielles elles-mêmes et pour l'articulation entre ces approches et l'apprentissage de compétences langagières de communication dans des langues particulières (articulation conceptuelle et pratique, dans les curricula, dans la classe) ainsi que, plus largement, pour l'articulation entre les apports des approches plurielles et ceux de disciplines non linguistiques. Par-delà, on peut considérer que ce référentiel, qui s'inscrit dans la tradition de ce qu'il est convenu d'appeler «référentiel de compétences» peut contribuer à la reconnaissance même de l'intérêt de ces approches, dont le potentiel n'est pas mis en évidence (de sorte que deux d'entre elles, l'éveil aux langues et l'intercompréhension entre langues parentes, sont souvent perçues comme de simples «sensibilisations»).

INTERVIEWER: Et maintenant où en êtes-vous dans le projet CARAP? Quels sont vos projets d'avenir?

MICHEL CANDELIER: Grâce au soutien du Centre européen pour les langues vivantes (CELV) nous avons pu développer un site web (http://carap.ecml.at/CARAP/tabid/2332/language/fr-FR/Default.aspx) qui donne accès au référentiel et offre une présentation dynamique des descripteurs de ressources (savoirs, savoirêtre et savoir-faire) qui composent le référentiel. On y trouve aussi un accès à une représentation des descripteurs qui les situe dans un cursus d'apprenant. Le site comprend une base de données de matériaux didactiques susceptibles de favoriser le développement en classe des savoirs, savoirêtre et savoir-faire du référentiel. L'enseignant peut les choisir en fonction des savoirs, savoir-être et savoir-faire qu'il a repérés parmi les descripteurs retenus par le CARAP, mais aussi en fonction du niveau de l'enseignement qui le concerne, des langues dont il désire traiter... et même des thématiques qui lui semblent pouvoir motiver ses élèves..

Le CELV va prochainement financer des projets de deux ans qui vont permettre de mieux diffuser le CARAP et de développer l'intérêt des parents pour les approches plurielles et l'éducation plurilingue.

INTERVIEWER: Je voudrais vous remercier pour votre temps et pour toutes ces informations que vous avez partagées avec nous pendant cet entretien. 


\section{Références Auteur:}

Xavier Pascual Calvo est docteur expert en éducation interculturelle, membre du groupe de recherche GREIP en enseignement et intéraction plurilingues, membre expert du CELV de Graz et professeur de langues étrangères à

l'Institut Obert de Catalunya.

Email: xpcbarcelona@gmail.com

Pour citer cet article:

Pascual Calvo, X. (2012). Les approches plurielles dans l'enseignement des langues: un entretien avec Michel

Candelier. Bellaterra Journal of Teaching \& Learning Language \& Literature, 5(1), 96-103. 